\title{
Influência da altura do salto de sapatos na função venosa da mulher jovem
}

\author{
Influence of shoe heel height on venous function in young women \\ Wagner Tedeschi Filho ${ }^{1}$, Carlos Eli Piccinato², Takachi Moriya ${ }^{3}$, Edwaldo E. Joviliano ${ }^{4}$, \\ Nei R. A. Dezotti ${ }^{4}$
}

\section{Resumo}

Contexto: A influência da altura do salto de sapatos na função venosa é ainda assunto controverso na literatura mundial. A importância da ergonomia na qualidade de vida é um fator consagrado e situações que a prejudiquem como permanência prolongada na posição supina, qualidade dos calçados e condições do local de trabalho podem interferir na saúde do indivíduo.

Objetivo: Estudar a influência da altura do salto do sapato na drenagem venosa dos membros inferiores, utilizando-se a pletismografia a ar (PGA).

Método: Quinze mulheres, com idade média de 24,6 anos, assintomáticas, utilizando calçados de tamanhos apropriados, foram examinadas em três momentos: descalças $(0 \mathrm{~cm})$, salto médio $(3,5 \mathrm{~cm})$ e alto $(7 \mathrm{~cm})$. Apresentavam índice de massa corporal $<25 \mathrm{e}$ foram classificadas de acordo com a Classificação Internacional CEAP, em critérios: clínico ( $\mathrm{C} 0$ ou $\mathrm{C} 1)$, etiológico (Ep), anatômico (As) e fisiopatológico (Pr). Os valores do índice de enchimento venoso (IEV), da fração de ejeção (FE) e da fração de volume residual (FVR) foram separados em três categorias pela altura do salto e comparados entre si, utilizando-se a análise de variância para médias repetidas (ANOVA).

Resultados: Houve diminuição da FE e aumento da FVR no grupo de salto alto em relação ao grupo descalço $(\mathrm{p}<0,005)$. Não ocorreu diferença desses parâmetros entre o grupo de salto médio e os outros grupos. O IEV comportou-se de maneira semelhante nas três situações avaliadas.

Conclusão: $O$ salto alto diminui a função de bomba muscular demonstrado pela queda da FE e aumento da FVR, podendo, com o seu uso contínuo, provocar hipertensão venosa nos membros inferiores, o que poderia ser preditivo de sintomatologia na doença venosa.

Palavras-chave: Pletismografia, ergonomia, membros inferiores, insuficiência venosa.

\begin{abstract}
Background: The influence of shoe heel height on venous function is still a controversial subject in the international literature. The importance of ergonomics for quality of life is a universally accepted factor, and situations that impair it, such as prolonged permanence in the supine position, shoe quality and workplace conditions may interfere with the individual's health.
\end{abstract}

Objective: To analyze the influence of shoe heel height on lower limb venous drainage using air plethysmography.

Method: Fifteen asymptomatic women with mean age of 24.6 years, wearing shoes of appropriate size were examined in three different situations: barefoot $(0 \mathrm{~cm})$, medium heels $(3.5 \mathrm{~cm})$ and high heels $(7 \mathrm{~cm})$. Body mass index was $<25$ and the subjects were classified according to the CEAP International Classification based on clinical (C0 or $\mathrm{C} 1$ ), etiologic (Ep), anatomic (As) and physiopathological (Pr) criteria. The values of venous filling index (VFI), ejection fraction (EF) and residual volume fraction (RVF) were divided into three categories according to heel height and compared to one another by repeated means analysis of variance (ANOVA).

Results: EF was decreased and RVF was increased in the high heel group compared to the barefoot group $(p<0.005)$. These parameters did not differ between the medium heel group and the other groups. VFI showed a similar behavior in the three situations evaluated.

Conclusion: High heels reduce muscle pump function, as demonstrated by the fall in EF and increase in RVF, and their continued use may provoke venous hypertension in the lower limbs, possibly representing a predictive factor of venous disease symptoms.

Keywords: Plethysmography, ergonomics, lower limbs, venous insufficiency.

1. Médico residente, Disciplina de Cirurgia Vascular e Angiologia, Hospital das Clínicas, Faculdade de Medicina de Ribeirão Preto, Universidade de São Paulo (USP), Ribeirão Preto, SP.

2. Professor titular e Chefe da Disciplina de Cirurgia Vascular e Angiologia, Hospital das Clínicas, Faculdade de Medicina de Ribeirão Preto, USP, Ribeirão Preto, SP.

3. Doutor. Professor, Disciplina de Cirurgia Vascular e Angiologia, Hospital das Clínicas, Faculdade de Medicina de Ribeirão Preto, USP, Ribeirão Preto, SP.

4. Doutor em Cirurgia. Médico assistente, Disciplina de Cirurgia Vascular e Angiologia, Hospital das Clínicas, Faculdade de Medicina de Ribeirão Preto, USP, Ribeirão Preto, SP.

Trabalho realizado na Disciplina de Cirurgia Vascular, Departamento de Cirurgia e Anatomia, Faculdade de Medicina de Ribeirão Preto, Universidade de São Paulo (USP), Ribeirão Preto, SP. Apresentado e premiado em 20 lugar no IX Panamerican Congress on Vascular and Endovascular Surgery, realizado na cidade do Rio de Janeiro, RJ, no período de 31 de outubro a 04 de novembro de 2006.

Artigo submetido em 08.05.07, aceito em 28.08.07.

J Vasc Bras. 2007;6(4):352-358.

Copyright $\odot 2007$ by Sociedade Brasileira de Angiologia e de Cirurgia Vascular 


\section{Introdução}

O salto alto, difundido entre as mulheres por seu valor estético, deixa de ser exclusividade dos momentos especiais ou de gala para ser opção diária no ambiente de trabalho.

Variações na forma, consistência e material usado na fabricação do calçado, principalmente a altura do salto, podem ter influência na saúde da mulher. Voluntárias que usaram solados macios depois do uso de solados rígidos apresentaram não só maior conforto referido mas também redução no edema e no impacto calcâneo ao final de apenas $2 \mathrm{~h}$ de uso ${ }^{1}$.

A ergonomia e condições de trabalho, referentes à postura (sentada, de pé e caminhando), tipo de calçado e dureza do solo, influem nos sintomas de cansaço, dor lombar e plantar. Essas queixas foram objetivamente confirmadas a partir de dados eletromiográficos das pernas e da musculatura lombar paravertebral e por meio de sensores de pressão e impacto instalados nos calçados ${ }^{1-3}$.

A pletismografia a ar (PGA) foi utilizada para evidenciar a correspondência das queixas de dor e fadiga em membros inferiores de trabalhadores que usavam calçados rígidos e em permanência ortostática prolongada, demonstrando que as queixas de fadiga têm associação com o aumento do volume das pernas ${ }^{4}$. A espessura e dureza do salto também têm implicações ortopédicas, como na formação de esporões calcâneos ${ }^{5}$. Mas nem tudo no âmbito da saúde dos membros inferiores limita-se ao calçado usado, característica do solo ou à posição do indivíduo: a velocidade da marcha, quando aumentada, eleva os pico de pressão plantar ${ }^{6}$, e a constituição biofísica também interfere nas queixas de dor e fadiga, pois são mais comuns em indivíduos mais baixos ou com maior massa ${ }^{7}$.

Apesar do conhecimento disponível, não se encontrava na literatura informações específicas sobre a influência dos calçados com saltos elevados na drenagem venosa dos membros inferiores.

Desta forma, objetivou-se neste trabalho investigar a influência da altura dos saltos de sapatos $(3,5$ e $7 \mathrm{~cm})$ na drenagem venosa dos membros inferiores da mulher jovem em relação à posição descalça, utilizando-se a PGA.

\section{Método}

Foram estudadas 15 mulheres assintomáticas na faixa etária de 20 a 30 anos, cuja média foi 24,6 anos. Apresentavam índice de massa corporal $<25$ e foram classificadas de acordo com a Classificação Internacional CEAP, utilizada como padrão no estudo da DVC, em critério clínico ( $\mathrm{C} 0$ ou $\mathrm{C} 1)$, critério etiológico (Ep), critério anatômico (As) e critério fisiopatológico $(\mathrm{Pr})^{8}$.

Os exames foram realizados no Laboratório de Investigação Vascular não-Invasiva da Disciplina de Cirurgia Vascular do Hospital das Clínicas da Faculdade de Medicina de Ribeirão Preto da Universidade de São Paulo, no período vespertino, por dois examinadores. As manobras foram iniciadas após demonstração do exame à voluntária e após compreensão e confiança da mesma.

A PGA foi realizada em três momentos com cada voluntária: descalças $(0 \mathrm{~cm})$, salto médio $(3,5 \mathrm{~cm})$ e salto alto $(7 \mathrm{~cm})$. Todas utilizaram calçados de tamanho apropriado e assinaram termo de consentimento livre e esclarecido aprovado pelo Comitê de Ética em Pesquisa do Hospital das Clínicas da Faculdade de Medicina de Ribeirão Preto da Universidade de São Paulo, Processo n. 15.309/2005.

A função da bomba muscular da panturrilha foi estudada pela PGA. O aparelho utilizado foi o SDV 3000 da marca Angiotec ${ }^{\circledR}$ (Belo Horizonte, Brasil), com calibração automatizada por computador.

Para realização do exame, a temperatura do laboratório foi mantida entre 22 e $24{ }^{\circ} \mathrm{C}$. Utilizou-se maca de exame a uma altura próxima dos $55 \mathrm{~cm}$, para facilitar a mobilização do paciente da posição supina para ortostática.

O PGA consiste em um aparelho, conectado através de cabos a um computador, e de uma mangueira de poliuretano, com 2,5 $\mathrm{m}$ de comprimento, conectada a um manguito com $35 \mathrm{~cm}$ de comprimento e aproximadamente $5 \mathrm{~L}$ de capacidade, do mesmo material que a mangueira.

O exame foi realizado com o paciente em posição supina, para o esvaziamento das veias, mantendo a perna elevada a $45^{\circ}$ e o joelho levemente fletido. O pé era apoiado pelo calcanhar em um suporte a uma altura de 
$20 \mathrm{~cm}$ em relação à mesa de exame. Colocava-se na perna do paciente um manguito pneumático de tamanho apropriado, envolvendo toda a extensão da perna, desde o joelho até o tornozelo, não excedendo os limites da perna, permitindo assim um bom contato do manguito com a pele. Este, por sua vez, promovia mínima oclusão das veias superficiais.

Iniciava-se, assim, a calibragem do aparelho de forma automática. Cuidados foram tomados para que o manguito não ficasse em contato com o suporte de elevação do pé e com a mesa de exame, para não alterar a leitura feita pelo aparelho e, conseqüentemente, o resultado do exame.

Após comando eletrônico, o manguito era inflado automaticamente a uma pressão selecionada de 6 $\mathrm{mmHg}$, transmitindo ao transdutor de pressão, localizado no aparelho, amplificando o sinal e registrando-o na tela do computador, em forma de gráfico.

Um valor basal de volume era obtido e então solicitava-se ao paciente que levantasse a perna com auxílio do examinador, apoiando-se, na posição de pé, no membro não avaliado. Em um andador, o paciente apoiava as mãos para sua melhor sustentação. $\mathrm{O}$ aumento do volume da perna era observado até chegar a um platô, indicando que as veias estavam cheias. A diferença entre o volume inicial e o volume no platô representava o volume venoso funcional (VV).

$\mathrm{Na}$ seqüência, solicitava-se ao paciente que se apoiasse com os dois pés no chão e realizasse movimento de flexão plantar, provocando a contratura da musculatura da panturrilha, retomando a seguir à posição inicial de repouso ou relaxamento do membro avaliado. A queda que se observava na curva do gráfico corresponde ao volume ejetado (VE), resultado da contração muscular da panturrilha.

Após esse movimento, um novo platô era atingido, geralmente mais elevado que o inicial e solicitava-se ao paciente realizar 10 movimentos de flexão plantar, numa velocidade de um movimento por segundo. O decréscimo de volume observado era também anotado. $\mathrm{O}$ volume residual (VR) era calculado a partir do valor basal final em relação ao volume restante ao término dos movimentos.

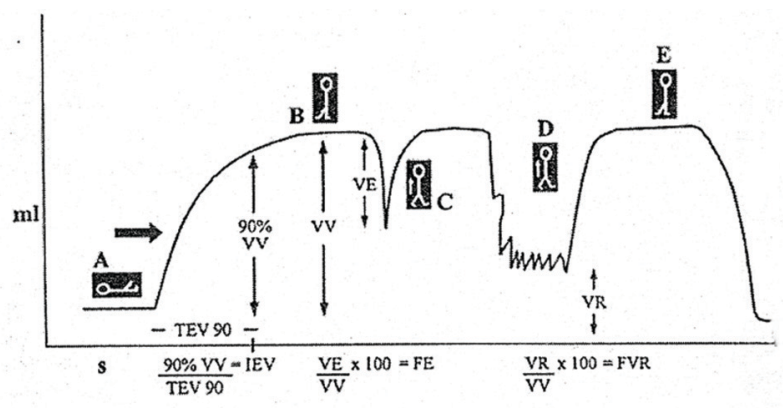

$90 \% \mathrm{VV}=90 \%$ do volume venoso $\mathrm{A}=$ voluntária deitada $; \mathrm{B}=$ voluntária em ortostase 10 vezes; $\mathrm{E}=$ ortostase novamente; $\mathrm{FE}=$ fração de ejeção; FVR = fração de volume residual; IEV = índice de enchimento venoso; TEV $90=$ tempo de $90 \%$ do enchimento venoso; $\mathrm{VE}=$ volume ejetado; $\mathrm{VR}=$ volume residual; $\mathrm{VV}=$ volume venoso funcional.

Figura 1 - Exemplo de A) curva normal e B) as posições das voluntárias na obtenção de cada dado; C) voluntária realizando um movimento de elevação na ponta dos pés, D) voluntária repetindo o valor ${ }^{9}$

Após o término das flexões plantares, solicitava-se ao paciente retomar a posição de repouso e relaxar a perna examinada, até atingir um novo platô de enchimento venoso. Findo o nivelamento, com auxílio do examinador, o paciente retomava à posição supina, na mesa de exame, com o pé sobre o suporte, observando-se, no gráfico o esvaziamento total do membro, mostrado por um nivelamento do gráfico, correspondendo ao volume final (VF).

O gráfico volume/tempo era visualizado na tela do computador. Uma vez traçado o gráfico, eram feitas marcações com cursor do programa do computador, correspondente ao volume inicial (ZERO), volume venoso (VV), volume ejetado (VE), volume de reenchimento (VRe), volume residual mínimo (VR min) e, por fim, o volume final (VF). O programa marcava automaticamente o tempo gasto para alcançar $90 \%$ do volume venoso $(90 \% \mathrm{VV})$ (Figura 1).

Realizadas as marcações no gráfico, o computador processava os cálculos automaticamente para índice de enchimento venoso (IEV), fração de ejeção (FE) e fração do volume residual (FVR) ${ }^{9}$.

Finalizado o procedimento descrito acima com a voluntária descalça, a mesma rotina era repetida com calçados em ambos os pés, primeiro com salto de $3,5 \mathrm{~cm}$ 
e, após, com salto de $7 \mathrm{~cm}$, obtendo-se portanto três gráficos por voluntária. O manguito, de forma padrão, foi sempre instalado na perna esquerda.

O IEV é a variação de volume produzida no membro dividida pelo tempo correspondente em segundos, na passagem da posição deitada para a posição ortostática, calculado mediante a fórmula IEV $=90 \%$ x VV / TEV 90 e expresso em mL/s.

Estudou-se a função de bomba da panturrilha por meio dos dados obtidos pelas variáveis FE e FVR.

A FE fornece uma estimativa do retorno venoso durante a atividade física e de alterações que resultem em menos sangue ejetado. A FE é a porcentagem do volume venoso total, ejetada em uma única contração da musculatura da panturrilha, sendo calculada pela fórmula $\mathrm{FE}=(\mathrm{VE} / \mathrm{VV}) \times 100$ onde, VE é o volume ejetado e $\mathrm{VV}$, o volume venoso.

A FVR correlaciona-se linearmente com a pressão venosa ambulatorial (PVA), medindo-a de forma nãoinvasiva, ou seja, medida pela fórmula $F V R=(V R /$ VV) x 100 , onde VR é o volume residual ${ }^{10}$.

Os valores de literatura considerados normais ${ }^{11}$ são IEV $<2$, FE $>40 \%$ e FVR $<35 \%$.

Para comparar os valores das variáveis IEV, FE e FVR entre os saltos plano, médio e alto foi utilizada a análise de variância para médias repetidas (ANOVA) considerando nível de significância $\mathrm{p}<0,05$ pelo teste post hoc de Bonferroni ${ }^{12}$.

\section{Resultados}

Os valores do IEV, da FE e da FVR foram separados em três categorias pela altura do salto: descalças (0 $\mathrm{cm})$, salto médio $(3,5 \mathrm{~cm})$ e salto alto $(7 \mathrm{~cm})$. Para facilitar a leitura e compreensão dos resultados, esses valores estão apresentados em tabelas e figuras. Houve diminuição da drenagem venosa demonstrada pela diminuição da FE (Figura 2 e Tabela 1) aumento da FVR (Figura 3 e Tabela 2) no grupo de salto alto $(7 \mathrm{~cm}) \mathrm{em}$ relação ao grupo descalço $(0 \mathrm{~cm})$.

Não houve diferença significativa destes parâmetros entre o grupo de salto médio e os outros grupos, embora

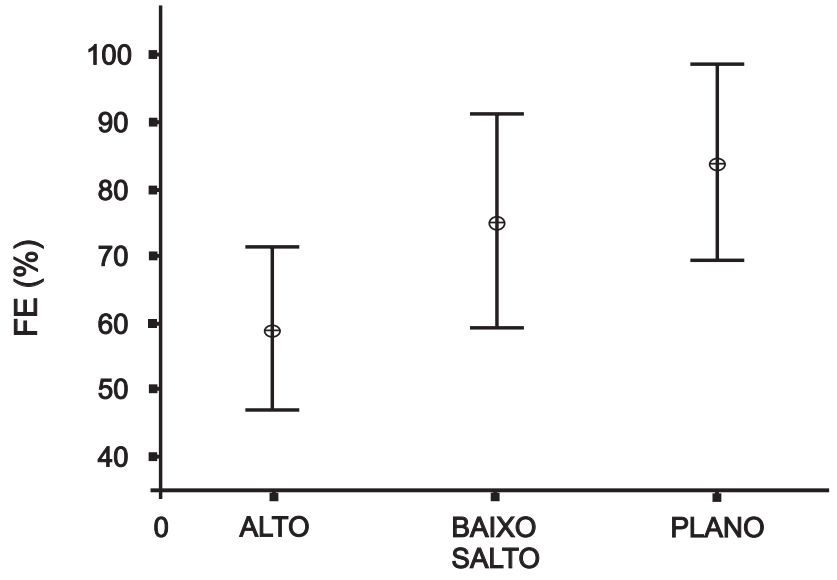

Figura 2 - Variação da fração de ejeção (FE) nas três situações do teste: descalço, salto baixo e salto alto de 15 mulheres jovens (20-30 anos)

tenha se estabelecido uma forte tendência de diminuição da FE e aumento da FVR nesse grupo em relação ao grupo descalço.

O IEV comportou-se de maneira semelhante nos três momentos estudados (Figura 4 e Tabela 3).

\section{Discussão}

A PGA é um método de grande valor na avaliação do sistema venoso dos membros inferiores, permitindo correlações clínicas e fisiológicas ${ }^{13}$. A PGA quantifica o volume venoso, o refluxo venoso superficial e profundo em mililitros por segundo, o grau de obstrução venosa e a capacidade de ejeção da bomba muscular da panturrilha de forma não-invasiva, pelos gráficos de volume sangüíneo adquiridos durante as manobras ${ }^{14}$.

Tabela 1 - Variação da fração de ejeção (FE) nas três situações do teste: descalço, salto baixo e salto alto

\begin{tabular}{|c|c|c|c|c|c|}
\hline FE & $\begin{array}{c}1^{\circ} \\
\text { quartil } \\
\text { (P25) }\end{array}$ & $\begin{array}{c}\text { Mediana } \\
\text { (P50) }\end{array}$ & $\begin{array}{c}3^{\circ} \\
\text { quartil } \\
\text { (P75) }\end{array}$ & Média & DP \\
\hline Descalço & 62,00 & 83,80 & 109,40 & 84,03 & 26,41 \\
\hline Baixo & 61,20 & 72,30 & 92,60 & 75,11 & 28,63 \\
\hline Alto & 40,40 & 57,10 & 75,50 & 59,08 & 21,67 \\
\hline
\end{tabular}

$\mathrm{DP}=$ desvio padrão; $\mathrm{FE}$ = fração de ejeção.

Como $\mathrm{p}=0,009$, há diferença significativa entre descalço, baixo e alto. Pelo teste post hoc de Bonferroni ${ }^{12}$, temos que a diferença significativa é entre descalço e alto, pois o descalço apresenta valores superiores ao alto $(\mathrm{p}=0,01)$. 


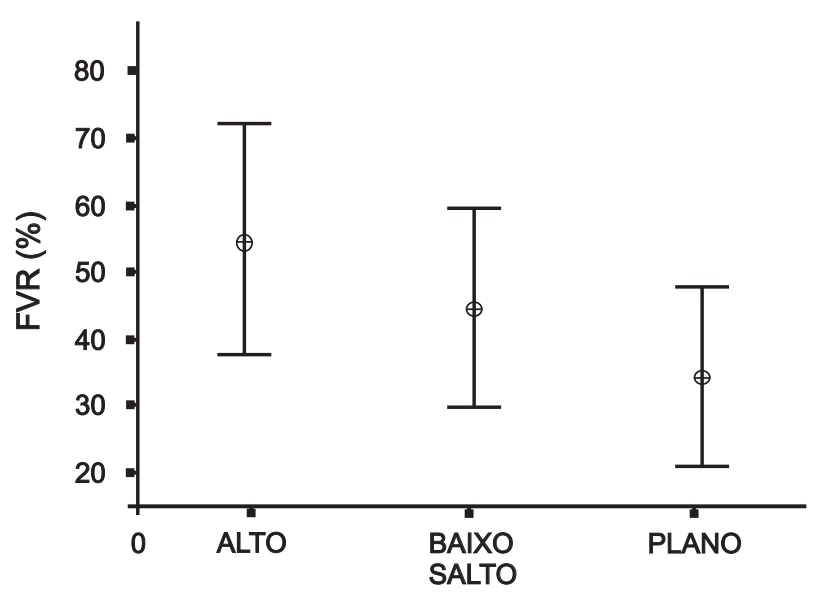

Figura 3 - Variação da fração de volume residual (FVR) nas três situações do teste: descalço, salto baixo e salto alto de 15 mulheres jovens (20-30 anos)

Tabela 2 - Variação da fração de volume residual (FVR) nas três situações do teste: descalço, salto baixo e salto alto

\begin{tabular}{lccccc}
\hline & $\begin{array}{c}\mathbf{1}^{\mathbf{0}} \\
\text { quartil } \\
\text { FVR }\end{array}$ & $\begin{array}{c}\text { Mediana } \\
(\mathbf{P} 25)\end{array}$ & $\begin{array}{c}\mathbf{3}^{\mathbf{0}} \\
\mathbf{q u a r t i l} \\
\mathbf{( P 7 5 )}\end{array}$ & Média & DP \\
\hline Descalço & 21,60 & 25,30 & 38,90 & 34,76 & 25,47 \\
Baixo & 28,40 & 33,80 & 58,90 & 44,55 & 27,41 \\
Alto & 22,80 & 59,10 & 81,40 & 56,19 & 31,17 \\
\hline
\end{tabular}

$\mathrm{DP}=$ desvio padrão; FVR = fração de volume residual. Como $\mathrm{p}=0,003^{*}$ há diferença significativa entre descalço, baixo e alto. Pelo teste post hoc de Bonferroni ${ }^{12}$, temos que a diferença significativa é entre descalço e alto, pois o descalço apresenta valores inferiores ao alto $(\mathrm{p}=0,01)$.

Segundo Yang, além dessas informações, a PGA pode revelar elementos orientadores de conduta terapêutica e avaliar o efeito do tratamento proposto ${ }^{15}$, inclusive caracterizando o estudo hemodinâmico durante o uso da compressão elástica ${ }^{16}$.

A avaliação da função bomba muscular da panturrilha de acordo com a idade ${ }^{17}$ e os efeitos do fortalecimento muscular da panturrilha na hemodinâmica venosa $^{18}$ foram observados, utilizando-se a PGA.

A FVR exprime o efeito combinado de refluxo venoso e da fração de ejeção com o exercício rítmico. Além disso, é um indicador relevante da pressão venosa

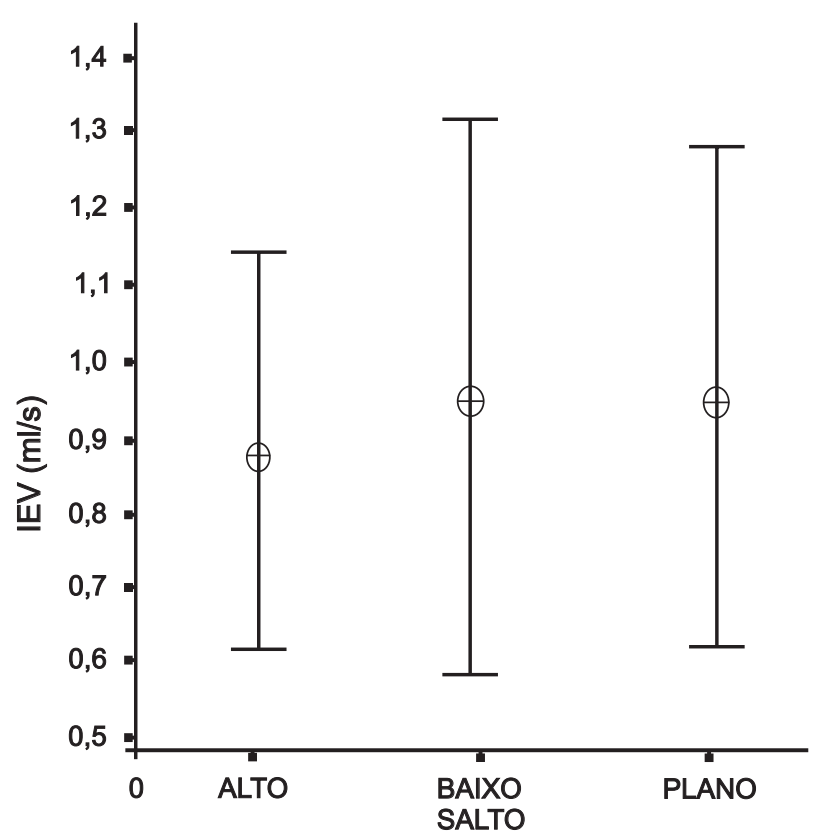

Figura 4 - Variação do índice de enchimento venoso (IEV) nas três situações do teste: descalço, salto baixo e salto alto de 15 mulheres jovens (20-30 anos)

Tabela 3 - Variação do índice de enchimento venoso (IEV) nas três situações do teste: descalço, salto baixo e salto

\begin{tabular}{lccccc}
\hline IEV & $\begin{array}{c}\mathbf{1}^{\mathbf{0}} \\
\text { quartil } \\
(\mathbf{P 2 5})\end{array}$ & $\begin{array}{c}\text { Mediana } \\
(\mathbf{P 5 0 )}\end{array}$ & $\begin{array}{c}\mathbf{3}^{\mathbf{q}} \\
\text { quartil } \\
\mathbf{( P 7 5 )}\end{array}$ & Média & DP \\
\hline Descalço & 0,44 & 0,81 & 1,28 & 0,95 & 0,59 \\
Baixo & 0,41 & 0,63 & 1,35 & 0,95 & 0,66 \\
Alto & 0,47 & 0,96 & 1,06 & 0,88 & 0,48 \\
\hline
\end{tabular}

$\mathrm{DP}=$ desvio padrão; IEV = índice de enchimento venoso. $\mathrm{p}=0,85$ não há diferença significativa entre os saltos descalço, baixo e alto.

ambulatorial e por isso foi um dos parâmetros escolhidos para avaliar a influência dos diferentes saltos de sapatos na hemodinâmica venosa ${ }^{19}$.

O principal mecanismo de retorno venoso dos membros inferiores é a ejeção do sangue através da contração muscular. A "bomba da panturrilha" é acionada a cada passo dado, ejetando o sangue contra a gravidade e, graças à competência valvar venosa, não há refluxo.

A mobilidade do tornozelo é fator essencial para a atuação eficiente da bomba da panturrilha. Quando se 
instala a anquilose tíbio-társica, produz-se a insuficiência venosa crônica irreversível, porque está anulada a função da bomba gêmeo-solear ${ }^{20}$. A contração da panturrilha durante a marcha tem com objetivo elevar o corpo na ponta dos pés, impulsionando-o. Aparentemente, o salto alto limita esse movimento de forma radical, diminuindo a ação da bomba. Quanto mais alto o salto, mais esse fenômeno é notado.

O grau de mobilidade do tornozelo em um indivíduo normal é ao redor de $70^{\circ}$, sendo $45^{\circ}$ às custas de flexão plantar e $25^{\circ}$ por extensão dorsal. Após estudar 32 membros de 26 adultos do sexo masculino, Back et al. ${ }^{21}$ concluíram que um grau limitado de mobilidade do tornozelo é um fator significativo no índice de ulceração venosa. O uso do salto, evidentemente, não é uma alteração definitiva, mas considerando o "tempo de exposição", diferentes níveis de prejuízo poderiam ocorrer.

Neste trabalho observou-se que não houve diferença do IEV nos três momentos (voluntário descalça, com salto $3,5 \mathrm{~cm} \mathrm{e} 7 \mathrm{~cm}$, respectivamente) avaliados, o que demonstra que a competência valvar global do sistema venoso dos membros inferiores manteve-se preservada durante as três fases do experimento, resultado que confirma o critério de seleção de não possuir doença venosa de qualquer espécie nas mulheres avaliadas.

A FE diminuiu com o uso do salto alto, o que pode ser explicado por um menor encurtamento das fibras musculares da panturrilha durante a sístole muscular. Conseqüentemente, o volume de sangue retido no membro aumenta.

O aumento da FVR com o uso de calçados de saltos mais elevados $(7 \mathrm{~cm})$, encontrado neste estudo, deve-se principalmente ao resíduo maior de sangue que permanece no membro após a queda da FE, considerando-se que a FVR é obtida na diástole muscular e representa a soma do volume venoso residual com o afluxo de sangue arterial que chega na panturrilha.

Apesar da impossibilidade de confirmar diferença significativa entre o grupo de salto médio $(3,5 \mathrm{~cm})$ e os outros grupos, estabeleceu-se uma forte tendência à diminuição da FE e aumento da FVR nesse grupo em relação ao grupo descalço. Provavelmente em estudos futuros, empregando-se casuística maior, tal tendência possa ser comprovada com significância estatística.

Conclui-se que o salto alto diminui a função de bomba muscular da panturrilha e que seu uso contínuo pode provocar situação de hipertensão venosa nos membros inferiores. Essa situação é componente primordial na evolução da doença venosa de estados de menor para maior gravidade clínica.

\section{Referências}

1. Hansen L, Winkel J, Jorgensen K. Significance of mat and shoe softness during prolonged work in upright position: based on measurements of low back muscle EMG, foot volume changes, discomfort and ground force reactions. Appl Ergon. 1998;29:217-24.

2. Krijnen RM, de Boer EM, Ader HJ, Bruynzeel DP. Venous insufficiency in male workers with a standing profession. Part 1: epidemiology. Dermatology. 1997;194:111-20.

3. Wakeling JM, Pascual SA, Nigg BM. Altering muscle activity in the lower extremities by running with different shoes. Med Sci Sports Exerc. 2002;34:1529-32.

4. Krijnen RM, de Boer EM, Ader HJ, Bruynzeel DP. Venous insufficiency in male workers with a standing profession. Part 2: diurnal volume changes of the lower legs. Dermatology. 1997;194:121-6.

5. Ozdemir H, Urguden M, Ozgorgen M, Gur S. [The relationship between the thickness and elasticity of the heel pad and heel pain]. Acta Orthop Traumatol Turc. 2002;36:423-8.

6. Drerup B, Hafkemeyer U, Moller M, Wetz HH. [Effect of walking speed on pressure distribution of orthopedic shoe technology]. Orthopade. 2001;30:169-75.

7. King PM. A comparison of the effects of floor mats and shoe in-soles on standing fatigue. Appl Ergon. 2002;33:477-84.

8. Porter JM, Moneta GL. Reporting standards in venous disease: an update. International Consensus Committee on Chronic Venous Disease. J Vasc Surg. 1995;21:635-45.

9. Dezotti NRA. Estudo da hemodinâmica venosa por meio da pletismografia a ar no pré e pós- operatório de varizes dos membros inferiores [tese]. Ribeirão Preto: USP-FMRP. 2006.

10. Criado E, Passman MA. Physiologic assessment of the venous system. In: Rutherford RB, editor. Vascular surgery. Philadelphia: WB Saunders; 2000. p. 165-91.

11. Evangelista SSM. Plestimografia no estudo das doenças venosas. In: Maffei FHA, Lastória S, Yoshida WB, Rollo HA. Doenças vasculares periféricas. Rio de Janeiro: MEDSI; 2002. p. 479-92.

12. Conover WJ. Practical nonparametric statistics. 2 ed. New York: John Wiley \& Sons; 1997.

13. Celestino RTS, Castro AA, Pitta GBB, Miranda Jr. F. Volume venoso nos membros inferiores em primigestas através da pletismografia a ar. J Vasc Bras. 2005;4:167-70.

14. Gaspar RA. Pletismografia a ar. J Suplência Vasc News. 2001;1:10. 
15. Yang D, Vandongen YK, Stacey MC. Variability and reliability of air plethysmographic measurements for the evaluation of chronic venous disease. J Vasc Surg. 1997;26:638-42.

16. Christopoulos D, Nicolaides AN, Cook A, Irvine A, Galloway JM, Wilkinson A. Pathogenesis of venous ulceration in relation to the calf muscle pump function. Surgery. 1989; 106:829-35.

17. Engelhorn CA, Beffa CV, Bochi G, Pullig RC, Picheth FS, Cunha SS. Avaliação por pletismografia a ar da função da bomba muscular da panturrilha conforme a idade. J Vasc Bras. 2003;2:13-6.

18. Lima RCM, Santiago L, Moura RMF, et al. Efeitos do fortalecimento muscular da panturrilha na hemodinâmica venosa $\mathrm{e}$ na qualidade de vida em um portador de insuficiência venosa crônica. J Vasc Bras. 2002;1:219-26.

19. Belczak CEQ. Responsabilidade das bombas músculo-venoarticulares distais na gênese de estase venosa. In: Thomaz JB, editor. Úlceras nos membros inferiores: diagnóstico e terapêutica. Fundo Editorial BYK; 2002. p. 93-103.
20. Canestri EA, Sánchez CF, Tropper UP. Tratado de flebologia y linfologia. Capítulo 4. In: Úrsula PT. Fisiologia y fisiopatologia. 2 ed. Buenos Aires: Fundación Flebológica; 1997. p. 24.

21. Back TL, Padberg FT Jr., Araki CT, Thompson PN, Hobson RW 2nd. Limited range of motion is a significant factor in venous ulceration. J Vasc Surg. 1995;22:519-23.

Correspondência:

Nei R. A. Dezotti

Faculdade de Medicina de Ribeirão Preto, Universidade de São Paulo

Campus USP, Bairro Monte Alegre

CEP 14048-900 - Ribeirão Preto, SP

Tel.: (16) 3602.2593

Fax: (16) 3620.1611

E-mail: neidezotti@yahoo.com.br

\section{ATENÇÃO}

\section{Registro de Ensaios Clínicos}

O Jornal Vascular Brasileiro apóia as políticas para registro de ensaios clínicos da Organização Mundial da Saúde (OMS) e do Internacional Committee of Medical Journal Editors (ICMJE; www. icmje.org), reconhecendo a importância dessas iniciativas para o registro e a divulgação internacional de informação sobre estudos clínicos, em acesso aberto. De acordo com essa recomendação, artigos de pesquisas clínicas devem ser registrados em um dos Registros de Ensaios Clínicos validados pelos critérios estabelecidos pela OMS e ICMJE, cujos endereços estão disponíveis no site do ICMJE:
www.actr.org.au
www.clinicaltrials.gov
www.isrctn.org
www.umin.ac.jp/ctr/index/htm
www.trialregister.nl

O número de identificação deverá ser registrado ao final do resumo.

A partir de 2008, somente serão aceitos para publicação artigos que tenham recebido um número de identificação nesses registros. 